\title{
Development of a real-time loop-mediated isothermal amplification method for the detection of severe fever with thrombocytopenia syndrome virus
}

\author{
Jae Woong Lee ${ }^{1}$, Yu-Jung Won ${ }^{1}$, \\ Lae Hyung Kang ${ }^{1}$, Sung-Geun Lee ${ }^{2}$, \\ Seung-Won Park ${ }^{3}$, and Soon-Young Paik ${ }^{1 \star}$ \\ ${ }^{1}$ Department of Microbiology, College of Medicine, \\ The Catholic University of Korea, Seoul 06591, Republic of Korea \\ ${ }^{2}$ Korea Zoonosis Research Institute, Jeonbuk National University, \\ Iksan 54596, Republic of Korea \\ ${ }^{3}$ Department of Biotechnology, Daegu Catholic University, \\ Gyeongsan-si 38430, Republic of Korea \\ (Received Mar 2, 2020 / Revised Apr 14, 2020 / Accepted Apr 16, 2020)
}

Severe fever with thrombocytopenia syndrome (SFTS) is being reported annually in South Korea since its first detection there in 2010. The causal agent is a negative-strand RNA virus $80-100 \mathrm{~nm}$ in diameter. It causes fever, thrombocytopenia, leukocytopenia, gastrointestinal symptoms, and neural symptoms. The mortality rate of SFTS was $32.6 \%$ among 172 cases reported from 2012 to 2015 in South Korea. Thus, is necessary to develop an effective diagnostic method that selectively identifies the isolates circulating in South Korea. The real-time reverse transcription loop-mediated isothermal amplification (RT-LAMP) assay is a simple, rapid, and sensitive approach for molecular diagnosis. Here, we designed novel primers for this assay and found that the technique had very high specificity, sensitivity, and efficiency. This real-time RTLAMP approach using the novel primers developed herein can be applied for early diagnosis of SFTSV strains in South Korea to reduce the mortality rate of SFTS.

Keywords: severe fever with thrombocytopenia syndrome (STFS), reverse transcription loop-mediated isothermal amplification (LAMP), molecular diagnostics, virus detection

\section{Introduction}

Since 2009, cases of acute febrile illness in the form of severe fever with thrombocytopenia syndrome (SFTS) have been reported in China (Yu et al., 2011). A new virus was isolated from these patients, which was identified to be SFTS bunyavirus (Yu et al., 2011). RNA sequence analysis showed that the virus belongs to a member of the Phlebovirus genus in the Bunyaviridae family (Yu et al., 2011). However, accord-

\footnotetext{
${ }^{*}$ For correspondence. E-mail: paik@catholic.ac.kr; Tel.: +82-2-2258-7342; Fax: +82-2-2258-7353

Copyright (C) 2020, The Microbiological Society of Korea
}

ing to the nomenclature by the International Committee of Taxonomy of Viruses (ICTV), SFTS virus (SFTSV) has been classified into the genus Banyangvirus, family Phenuiviridae (Takayama-Ito and Saijo, 2020). Surveillance data indicate that the incidence of SFTS is increasing throughout Asia, especially in China and Korea (Choi et al., 2016; Park et al., 2016; Wu et al., 2016; Zhang et al., 2017). The populations at the highest risk are farmers and elderly women living in rural farming communities and wooded and hilly areas, where immediate health care might not be available. In addition, human-to-human transmission of the virus through blood or close nosocomial contact has been reported (Xiong et al., 2012). Similar to other members of the family Phenuiviridae, SFTSV is a negative-strand RNA virus, and it comprises three single-stranded RNA genome segments designated as large (L), medium (M), and small (S) (Jiao et al., 2013). SFTSV is a spherical virion $80-100 \mathrm{~nm}$ in diameter, covered by a $5-7-$ nm-thick lipid bilayer envelope (Li, 2011; Lu et al., 2011; Yu et al., 2011; Xiong et al., 2012). SFTSV infection typically has four distinct phases: incubation, fever, multiple organ failure, and convalescence (Liu et al., 2014). SFTS patients exhibit fever, thrombocytopenia, leukocytopenia, gastrointestinal and neural symptoms, and bleeding tendency. The levels of liver and cardiac enzymes are commonly increased in SFTS patients, followed by damage in the liver and heart, respectively (Yu et al., 2011; Gai et al., 2012). SFTS was first reported in China in 2009, followed by Korea in 2012, and Japan in 2013. However, evidence of SFTS infections in South Korea from 2010 has been found, indicating that SFTS infections in South Korea occurred around the same time as reported in China (Kim et al., 2018). A total of 172 cases were reported in South Korea from 2012 to 2015, with a mortality rate of $32.6 \%$ (Choi et al., 2016). This mortality rate is higher than that recently reported in China (12.2\%); the first country where SFTS was reported (Guo et al., 2016). For early detection and diagnosis, quantitative real-time reverse transcription PCR (RT-PCR) and double-antigen sandwich enzymelinked immunosorbent assay (ELISA) have been used thus far. These methods detect a specific genetic region, which is the $S$ segment, because the $S$ segment is the most conserved gene. However, the $\mathrm{L}$ segment also has a conserved region and can be used as a target for the loop-mediated isothermal amplification (LAMP) method. There are no differences in sensitivity or specificity by the L, M, and S segments (Jiao et al., 2012; Sun et al., 2012; Yoshikawa et al., 2014; Baek et al., 2018). The RT-PCR and ELISA methods have limitations compared with the LAMP method, which is simpler and more rapid and sensitive for in vitro nucleic acid amplifica- 
tion (Yang et al., 2012; Huang et al., 2014). LAMP can detect very few copies of DNA under isothermal conditions. It is highly efficient, sensitive, and specific for target sequences. Moreover, LAMP is applicable to RNA upon use of reverse transcriptase and DNA polymerase (Notomi et al., 2000). Because of these advantages of the LAMP method, RT-LAMP has already been applied in the detection of several RNA viruses, such as enterovirus 71 , influenza $\mathrm{A} H 1 \mathrm{~N} 1$ virus, coronavirus, West Nile virus (WNV), and Ebola virus (Parida et al., 2004, 2011; Poon et al., 2005; Kurosaki et al., 2007; Ma et al., 2010; Jiang et al., 2011). In this study, we applied newly designed LAMP primers to target the L genes of SFTSV isolated in South Korea, and we determined the specificity, sensitivity, and efficiency of this approach.

\section{Materials and Methods}

\section{Virus samples and RNA extraction}

Jeju Halla General Hospital received written consent for sample collection from adult patients with SFTSV infection. The Korea Centers for Disease Control and Prevention (KCDC) standard protocol (conventional PCR) was used for detecting and confirming the virus as SFTSV. The Institutional Review Board reviewed and approved the use of the study samples for the purpose of research, as this study does not affect patients. All the experimental work and sample collections were supervised by the Catholic Medical Center Office of the Human Research Protection Program (CMC OHRP) of South Korea (approval number MC19SESI0015). The SFTSV CUK-JJ01 strain propagation, cell lines maintenances, and viral RNA extraction were performed as described in a published study (Won et al., 2019). In vitro experiments using SFTSV were conducted in a Bio Safety Level 3 (BSL-3) facility at the Korea Zoonosis Research Institute, Jeonbuk National University. The control RNA viruses were WNV, Japanese encephalitis virus (JEV), dengue viruses 2 and 3 (DENV-2, DENV-3), Zika virus, chikungunya virus, and influenza virus (H5N1). Their RNAs were extracted from $140 \mu \mathrm{l}$ of the viral stocks using QIAamp ${ }^{\circledR}$ Viral RNA Mini Kits (Qiagen, \#52906) according to the manufacturer's instructions.

\section{Design of primers for real-time RT-LAMP}

A total of 44 genomic sequences of different strains of SFTSV were retrieved from GenBank (https://www.ncbi.nlm.nih.gov/ genbank/) and analyzed using CLC Main Workbench 7 (version 7.6.4.) to identify conserved regions of the nucleotide sequence of SFTSV. Six SFTSV-specific RT-LAMP primers targeting the L gene were designed (Table 1). The primers consist of two external primers (forward outer primer F3 and backward outer primer B3), two internal primers (forward inner primer FIP and backward inner primer BIP), and two loop primers (forward loop primer LoopF and backward loop primer LoopB). The reaction was carried out at $65^{\circ} \mathrm{C}$ for 50 min using a WarmStart LAMP Kit (NEB, \#E1700).

\section{Conventional RT-PCR and real-time RT-PCR primer de-} sign and amplification

The Qiagen One-Step RT-PCR Kit (Qiagen, \#210212) was used for conventional RT-PCR, using the primers NP2-F (5'-CATCATTGTCTTTGCCCTGA-3') and NP2-R (5'-A GAAGACAGAGTTCACAGCA-3') (Oh et al., 2016). Reverse transcription reactions were incubated at $50^{\circ} \mathrm{C}$ for $30 \mathrm{~min}$, followed by $95^{\circ} \mathrm{C}$ for $15 \mathrm{~min}$. The amplification was performed according to the following conditions: $30 \mathrm{sec}$ at $94^{\circ} \mathrm{C}, 30 \mathrm{sec}$ at $52^{\circ} \mathrm{C}$, and $1 \mathrm{~min}$ at $72^{\circ} \mathrm{C}$ for 35 cycles, followed by a final extension step at $72^{\circ} \mathrm{C}$ for $10 \mathrm{~min}$. The $\mathrm{iTaq}^{\mathrm{TM}}$ Universal SYBR $^{\circledR}$ Green One-Step Kit (Bio-Rad, \#172-5150) was used for real-time RT-PCR. The primers $5^{\prime}$-GGGTCCCTGAAG GAGTTGTAAA-3' and 5' -TGCCTTCACCAAGACTATC AATGT- $3^{\prime}$ were designed to target conserved sequences in the $\mathrm{S}$ segment of this virus (Cui et al., 2012). Real-time RTPCR cycling was performed on a Bio-Rad ${ }^{\circledR}$ CFX $96^{\mathrm{TM}}$ system as follows: reverse transcription reaction at $50^{\circ} \mathrm{C}$ for $10 \mathrm{~min}$, polymerase activation at $95^{\circ} \mathrm{C}$ for $1 \mathrm{~min}$, and amplification under 40 cycles of denaturation at $95^{\circ} \mathrm{C}$ for $10 \mathrm{sec}$ and annealing/extension at $60^{\circ} \mathrm{C}$ for $30 \mathrm{sec}$.

\section{Synthesis of RNA transcripts for testing the sensitivity and efficiency}

Synthesis of RNA transcripts and calculation of copy numbers were performed according to previous studies (Devonshire et al., 2011; Lee et al., 2011). RNA transcripts were prepared for three types of assays: conventional RT-PCR, real-time RTPCR, and real-time RT-LAMP, each containing the necessary primer sequence information. Serial 10-fold dilutions of RNA transcripts with titers from $5 \times 10^{9}$ copies to $5 \times 10^{1}$ copies were used as templates. We evaluated the sensitivity and efficiency of the three assays.

\section{Results}

\section{Specificity of the real-time RT-LAMP assay}

For the specificity test of the real-time RT-LAMP assay, we used extracted viral RNA as the template. The results showed

\begin{tabular}{clr} 
Table 1. Primer design & & \\
\hline Primer & & \multicolumn{1}{c}{ Sequence $\left(5^{\prime}-3^{\prime}\right)$} \\
\hline F3 & TGGTACATTGATGCTGTGG & $2678-2696$ \\
B3 & TCCACTTCTCGGCATCA & $2968-2984$ \\
FIP & CTCTGAGGCCACCATGCTGGAAGGCAGTTCTAGATGACG & $2758-2778,2709-2726$ \\
BIP & CCACACGAGACTGTTGCCAGGCTGACTTCAGTCCATG & $2858-2876,2909-2926$ \\
LoopF & TTGAAGAGACAGATTCGCATG & $2731-2751$ \\
LoopB & CTAGGCTCAAGAATTCCATCATAGA & $2880-2904$ \\
\hline
\end{tabular}

${ }^{a}$ Genome position according to the strain's complete sequence (GenBank accession number MH937374). 


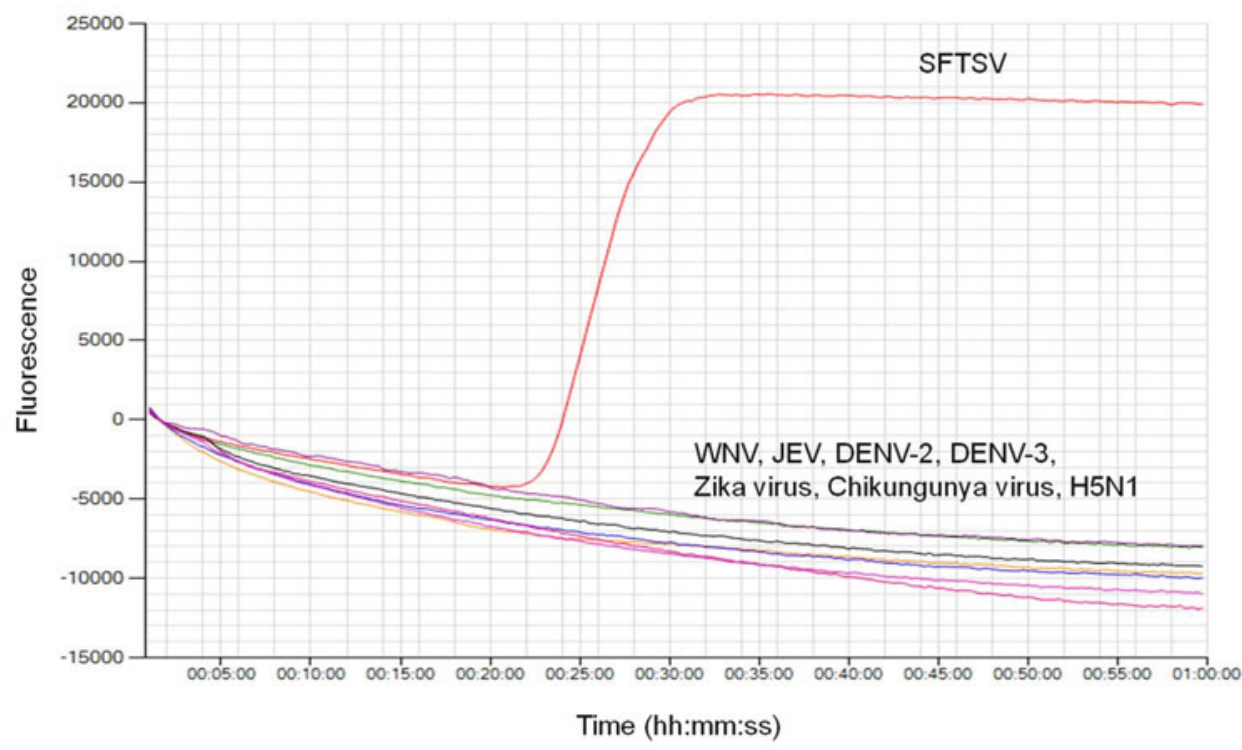

(A)
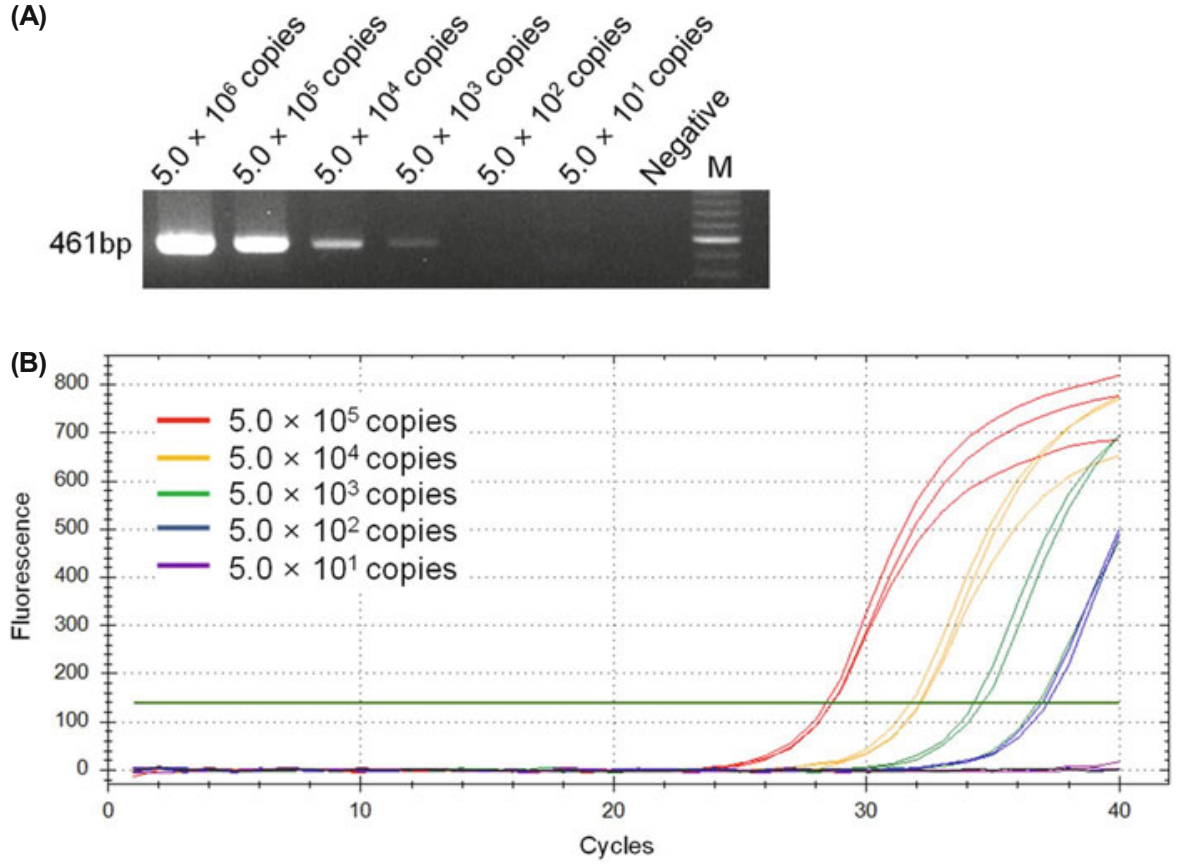

(C)

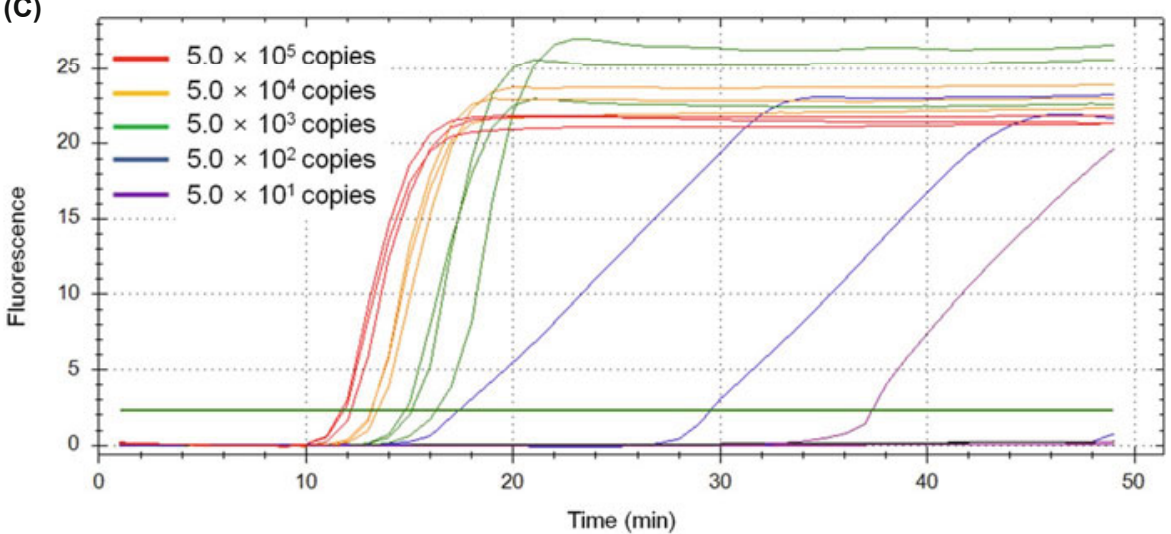

Fig. 1. Specificity of the real-time RTLAMP assay for SFTSV. Except SFTSV, no other unspecific products were amplified by the real-time RT-LAMP assay. Control viruses: West Nile virus (WNV), Japanese encephalitis virus (JEV), dengue viruses (DENV-2 and DENV-3), Zika virus, chikungunya virus, and influenza virus (H5N1).

Fig. 2. SFTSV detection efficiency with different assays. SFTSV detection efficiencies were tested using dilutions of SFTSV RNA transcripts ranging from copy numbers of $5 \times 10^{6}$ to $5 \times 10^{1}$. (A) Agarose gels of conventional RT-PCR. The detection limit of the assay was $5 \times$ $10^{3}$ copies of RNA transcripts. M, molecular weight marker; Negative, negative control. (B) Real-time RT-PCR assay for SFTSV RNA transcripts. The detection limit was $5 \times 10^{2}$ copies of RNA transcripts. (C) Real-time RT-LAMP assay for SFTSV RNA transcripts. The detection limit was $5 \times 10^{2}$ copies of RNA transcripts. 
positivity for SFTSV, while WNV, JEV, DENV-2, DENV-3, Zika virus, chikungunya virus, or $\mathrm{H} 5 \mathrm{~N} 1$ were not amplified (Fig. 1). Thus, real-time RT-LAMP can detect SFTSV with high specificity.

\section{SFTSV detection efficiency}

The SFTSV detection efficiencies of conventional PCR, realtime RT-PCR, and real-time RT-LAMP assays were determined using dilutions of SFTSV RNA transcripts ranging from $5 \times 10^{6}$ copies to $5 \times 10^{1}$ copies. The results confirmed that all PCR assays detected at least $5 \times 10^{2}$ copies to $5 \times 10^{3}$ copies of SFTSV RNA genes (Fig. 2). However, the efficiency of real-time RT-LAMP was higher because it was faster than the other two PCR assays. Real-time RT-LAMP determined positive results in 11 to $16 \mathrm{~min}$ compared with real-time RTPCR, which determined positive results in 28 to 36 cycles with the same SFTSV RNA transcript copy number. Conventional RT-PCR takes at least $2 \mathrm{~h}$ to yield results on agarose gels, while real-time RT-PCR takes at least 30-40 min to obtain results. However real-time RT-LAMP can show the result in as little as 10-15 min with the same template conditions.

\section{Discussion}

SFTS is caused by SFTSV, which is a novel Banyangvirus of the Phenuiviridae family. Since 2009, more than 3,500 SFTS cases have been reported in China, Korea, and Japan ( $\mathrm{Li}$, 2015). The mortality rate for SFTS in South Korea was $47 \%$ in 2013 and $27 \%$ in 2015 (Park et al., 2016). More efficient diagnostic methods are required to further reduce this mortality rate. The conventionally used methods for diagnosis are ELISA and quantitative real-time RT-PCR (Jiao et al., 2012; Sun et al., 2012). However, these methods have limitations such as being time-consuming and having low accuracy (Baek et al., 2018). PCR-based molecular biological techniques have been widely adopted in diagnosis, and among them, the LAMP method in particular has extremely high sensitivity and specificity (Zeng et al., 2018; Deng et al., 2019). The real-time RT-LAMP method is rapid, highly specific, sensitive, and efficient, and can yield results in real time. Recently, the RT-LAMP assay has been utilized for the diagnosis of RNA viral pathogens, such as a novel swine acute diarrhea syndrome coronavirus and Japanese yam mosaic virus (Notomi et al., 2000; Fukuta et al., 2003; Wang et al., 2018). In this study, novel primers targeting the highly conserved L gene were designed for a real-time RT-LAMP assay (Table 1). The real-time RT-LAMP assay with these primers showed very high accuracy and specificity for SFTSV, without the amplification of the other control viruses (Fig. 1). Moreover, the real-time RT-LAMP assay showed more efficient results compared with conventional RT-PCR and real-time RT-PCR. The efficiency of the real-time RT-LAMP assay was over twofold higher, from a copy number of $5 \times 10^{5}$ to $5 \times 10^{3}$, compared with real-time RT-PCR (Fig 2). Thus, real-time RTLAMP is a more rapid and efficient method than real-time RT-PCR. Previous research has shown slightly more sensitive results using conventional one-step RT-PCR and quantitative one-step RT-PCR methods. However these methods take more than $1.5 \mathrm{~h}$ and then need an additional step for confirmation. Our novel primers and LAMP method can confirm SFTSV in $20 \mathrm{~min}$ in real time (Yoshikawa et al., 2014). SFTS is an endemic disease with a high mortality rate in Asian countries, especially South Korea (Choi et al., 2016), warranting a novel primer set for real-time RT-LAMP to detect SFTSV isolates found in South Korea for early diagnosis and thus, a reduced mortality rate. Research groups in China and Korea have developed assays for SFTSV detection using real-time RT-LAMP with primers they designed to target S and L segments (Yang et al., 2012; Huang et al., 2014; Baek et al., 2018). However, we designed a novel primer set targeting a totally different highly conserved region of the L segment, for detecting SFTSV isolated in South Korea using LAMP. Our laboratory-based method is accurate and provides objective results that can be viewed in real time, enabling a simpler and more rapid and sensitive molecular diagnosis.

\section{Acknowledgments}

The present study was supported by a grant from the NextGeneration BioGreen21 Program (grant no. PJ011835), Rural Development Administration, Republic of Korea.

\section{Conflict of Interest}

The authors declare no conflict of interest.

\section{References}

Baek, Y.H., Cheon, H.S., Park, S.J., Lloren, K.K.S., Ahn, S.J., Jeong, J.H., Choi, W.S., Yu, M.A., Kwon, H.I., Kwon, J.J., et al. 2018. Simple, rapid and sensitive portable molecular diagnosis of stts virus using reverse transcriptional loop-mediated isothermal amplification (RT-LAMP). J. Microbiol. Biotechnol. 28, 1928-1936.

Choi, S.J., Park, S.W., Bae, I.G., Kim, S.H., Ryu, S.Y., Kim, H.A., Jang, H.C., Hur, J., Jun, J.B., Jung, Y., et al. 2016. Severe fever with thrombocytopenia syndrome in South Korea, 2013-2015. PLoS Negl. Trop. Dis. 10, e0005264.

Cui, L., Ge, Y., Qi, X., Xu, G., Li, H., Zhao, K., Wu, B., Shi, Z., Guo, X., Hu, L., et al. 2012. Detection of severe fever with thrombocytopenia syndrome virus by reverse transcription-cross-priming amplification coupled with vertical flow visualization. J. Clin. Microbiol. 50, 3881-3885.

Deng, M.H., Zhong, L.Y., Kamolnetr, O., Limpanont, Y., and Lv, Z.Y. 2019. Detection of helminths by loop-mediated isothermal amplification assay: a review of updated technology and future outlook. Infect. Dis. Poverty 8, 20.

Devonshire, A.S., Elaswarapu, R., and Foy, C.A. 2011. Applicability of RNA standards for evaluating RT-qPCR assays and platforms. BMC Genomics 12, 118 .

Fukuta, S., Iida, T., Mizukami, Y., Ishida, A., Ueda, J., Kanbe, M., and Ishimoto, Y. 2003. Detection of Japanese yam mosaic virus by RT-LAMP. Arch. Virol. 148, 1713-1720.

Gai, Z., Liang, M., Zhang, Y., Zhang, S., Jin, C., Wang, S.W., Sun, L., Zhou, N., Zhang, Q., Sun, Y., et al. 2012. Person-to-person transmission of severe fever with thrombocytopenia syndrome bunyavirus through blood contact. Clin. Infect. Dis. 54, 249-252.

Guo, C.T., Lu, Q.B., Ding, S.J., Hu, C.Y., Hu, J.G., Wo, Y., Fan, Y.D., 
Wang, X.J., Qin, S.L., Cui, N., et al. 2016. Epidemiological and clinical characteristics of severe fever with thrombocytopenia syndrome (SFTS) in China: an integrated data analysis. Epidemiol. Infect. 144, 1345-1354.

Huang, X.Y., Hu, X.N., Ma, H., Du, Y.H., Ma, H.X., Kang, K., You, A.G., Wang, H.F., Zhang, L., Chen, H.M., et al. 2014. Detection of new bunyavirus RNA by reverse transcription-loop-mediated isothermal amplification. J. Clin. Microbiol. 52, 531-535.

Jiang, T., Liu, J., Deng, Y.Q., Xu, L.J., Li, X.F., Han, J.F., Cao, R.Y., Qin, E.D., and Qin, C.F. 2011. Development and evaluation of a reverse transcription-loop-mediated isothermal amplification assay for rapid detection of enterovirus 71. J. Clin. Microbiol. 49, 870-874.

Jiao, L., Ouyang, S., Liang, M., Niu, F., Shaw, N., Wu, W., Ding, W., Jin, C., Peng, Y., Zhu, Y., et al. 2013. Structure of severe fever with thrombocytopenia syndrome virus nucleocapsid protein in complex with suramin reveals therapeutic potential. J. Virol. 87, 68296839.

Jiao, Y., Zeng, X., Guo, X., Qi, X., Zhang, X., Shi, Z., Zhou, M., Bao, C., Zhang, W., Xu, Y., et al. 2012. Preparation and evaluation of recombinant severe fever with thrombocytopenia syndrome virus nucleocapsid protein for detection of total antibodies in human and animal sera by double-antigen sandwich enzyme-linked immunosorbent assay. J. Clin. Microbiol. 50, 372-377.

Kim, Y.R., Yun, Y., Bae, S.G., Park, D., Kim, S., Lee, J.M., Cho, N.H., Kim, Y.S., and Lee, K.H. 2018. Severe fever with thrombocytopenia syndrome virus infection, South Korea, 2010. Emerg. Infect. Dis. 24, 2103-2105.

Kurosaki, Y., Takada, A., Ebihara, H., Grolla, A., Kamo, N., Feldmann, H., Kawaoka, Y., and Yasuda, J. 2007. Rapid and simple detection of Ebola virus by reverse transcription-loop-mediated isothermal amplification. J. Virol. Methods 141, 7883.

Lee, S.G., Lee, S.H., Park, S.W., Suh, C.I., Jheong, W.H., Oh, S., and Paik, S.Y. 2011. Standardized positive controls for detection of norovirus by reverse transcription PCR. Virol. J. 8, 260.

Li, D.X. 2011. Fever with thrombocytopenia associated with a novel bunyavirus in China. Chin. J. Exp. Clin. Virol. 25, 81-84.

Li, D.X. 2015. Severe fever with thrombocytopenia syndrome: a newly discovered emerging infectious disease. Clin. Microbiol. Infect. 21, 614-620.

Liu, Q., He, B., Huang, S.Y., Wei, F., and Zhu, X.Q. 2014. Severe fever with thrombocytopenia syndrome, an emerging tick-borne zoonosis. Lancet Infect. Dis. 14, 763-772.

Lu, J., Li, C., Zhang, F.S., Wu, W., Zhang, Q.F., Zhang, L., Wang, T., Wang, Q., Qiu, P.H., Liang, M.F., et al. 2011. Expression of structural and non-structural proteins of severe fever with thrombocytopenia syndrome bunyavirus. Chin. J. Virol. 27, 515-520.

Ma, X.J., Shu, Y.L., Nie, K., Qin, M., Wang, D.Y., Gao, R.B., Wang, M., Wen, L.Y., Han, F., Zhou, S.M., et al. 2010. Visual detection of pandemic influenza A H1N1 Virus 2009 by reverse-transcription loop-mediated isothermal amplification with hydroxynaphthol blue dye. J. Virol. Methods 167, 214-217.

Notomi, T., Okayama, H., Masubuchi, H., Yonekawa, T., Watanabe, K., Amino, N., and Hase, T. 2000. Loop-mediated isothermal amplification of DNA. Nucleic Acids Res. 28, E63.

Oh, S.S., Chae, J.B., Kang, J.G., Kim, H.C., Chong, S.T., Shin, J.H., Hur, M.S., Suh, J.H., Oh, M.D., Jeong, S.M., et al. 2016. Detection of severe fever with thrombocytopenia syndrome virus from wild animals and ixodidae ticks in the Republic of Korea. Vector Borne Zoonotic Dis. 16, 408-414.

Parida, M., Posadas, G., Inoue, S., Hasebe, F., and Morita, K. 2004. Real-time reverse transcription loop-mediated isothermal amplification for rapid detection of West Nile virus. J. Clin. Micro- biol. 42, 257-263.

Parida, M., Shukla, J., Sharma, S., Ranghia Santhosh, S., Ravi, V., Mani, R., Thomas, M., Khare, S., Rai, A., Kant Ratho, R., et al. 2011. Development and evaluation of reverse transcription loopmediated isothermal amplification assay for rapid and real-time detection of the swine-origin influenza A H1N1 virus. J. Mol. Diagn. 13, 100-107.

Park, S.W., Ryou, J., Choi, W.Y., Han, M.G., and Lee, W.J. 2016. Epidemiological and clinical features of severe fever with thrombocytopenia syndrome during an outbreak in South Korea, 20132015. Am. J. Trop. Med. Hyg. 95, 1358-1361.

Poon, L.L.M., Wong, B.W.Y., Chan, K.H., Ng, S.S.F., Yuen, K.Y., Guan, Y., and Peiris, J.S.M. 2005. Evaluation of real-time reverse transcriptase PCR and real-time loop-mediated amplification assays for severe acute respiratory syndrome coronavirus detection. J. Clin. Microbiol. 43, 3457-3459.

Sun, Y., Liang, M., Qu, J., Jin, C., Zhang, Q., Li, J., Jiang, X., Wang, Q., Lu, J., Gu, W., et al. 2012. Early diagnosis of novel SFTS bunyavirus infection by quantitative real-time RT-PCR assay. $J$. Clin. Virol. 53, 48-53.

Takayama-Ito, M. and Saijo, M. 2020. Antiviral drugs against severe fever with thrombocytopenia syndrome virus infection. Front. Microbiol. 11, 150.

Wang, H., Cong, F., Zeng, F., Lian, Y., Liu, X., Luo, M., Guo, P., and Ma, J. 2018. Development of a real time reverse transcription loopmediated isothermal amplification method (RT-LAMP) for detection of a novel swine acute diarrhea syndrome coronavirus (SADS-CoV). J. Virol. Methods 260, 45-48.

Won, Y.J., Kang, L.H., Lee, S.G., Park, S.W., Han, J.I., and Paik, S.Y. 2019. Molecular genomic characterization of severe fever with thrombocytopenia syndrome virus isolates from South Korea. J. Microbiol. 57, 927-937.

Wu, H.C., Xu, X.P., Wu, C., Lu, Q.B., Ding, Z.Y., and Lin, J.F. 2016. Spatial analysis and prediction of severe fever with thrombocytopenia syndrome in Zhejiang province, 2011-2015. Chin. J. Epidemiol. 37, 1485-1490.

Xiong, W.Y., Feng, Z.J., Matsui, T., and Foxwell, A.R. 2012. Risk assessment of human infection with a novel bunyavirus in China. Western Pac. Surveill. Response J. 3, 69-74.

Yang, G., Li, B., Liu, L., Huang, W., Zhang, W., and Liu, Y. 2012. Development and evaluation of a reverse transcription loop-mediated isothermal amplification assay for rapid detection of a new SFTS bunyavirus. Arch. Virol. 157, 1779-1783.

Yoshikawa, T., Fukushi, S., Tani, H., Fukuma, A., Taniguchi, S., Toda, S., Shimazu, Y., Yano, K., Morimitsu, T., Ando, K., et al. 2014. Sensitive and specific PCR systems for detection of both Chinese and Japanese severe fever with thrombocytopenia syndrome virus strains and prediction of patient survival based on viral load. J. Clin. Microbiol. 52, 3325-3333.

Yu, X.J., Liang, M.F., Zhang, S.Y., Liu, Y., Li, J.D., Sun, Y.L., Zhang, L., Zhang, Q.F., Popov, V.L., Li, C., et al. 2011. Fever with thrombocytopenia associated with a novel bunyavirus in China. $N$. Engl. J. Med. 364, 1523-1532.

Zeng, F., Cong, F., Liu, X., Lian, Y., Wu, M., Xiao, L., Yuan, W., Huang, R., Ma, J., Guo, P., et al. 2018. Development of a real time loop-mediated isothermal amplification method for detection of Senecavirus A. J. Virol. Methods 261, 98-103.

Zhang, Y., Shen, S., Shi, J., Su, Z., Li, M., Zhang, W., Li, M., Hu, Z., Peng, C., Zheng, X., et al. 2017. Isolation, characterization, and phylogenic analysis of three new severe fever with thrombocytopenia syndrome bunyavirus strains derived from Hubei Province, China. Virol. Sin. 32, 89-96. 